\title{
SCUBA mapping of outer Galaxy protostellar candidates
}

\author{
B. Mookerjea ${ }^{1,2}$, G. Sandell ${ }^{3}$, J. Stutzki ${ }^{1}$, and J. G. A. Wouterloot ${ }^{4}$ \\ 1 KOSMA, I. Physikalisches Institut, Universität zu Köln, Zülpicher Strasse 77, 50937 Cologne, Germany \\ e-mail: bhaswati@tifr.res.in \\ 2 Department of Astronomy, University of Maryland, College Park, MD 20742, USA \\ 3 SOFIA-USRA, NASA Ames Research Center, MS N211-3, Moffett Field, CA 94035, USA \\ 4 Joint Astronomy Centre, 660 N. A'ohoku Place, University Park, Hilo, Hawaii 96720, USA
}

Received 14 May 2007 / Accepted 16 July 2007

\section{ABSTRACT}

\begin{abstract}
Aims. We aim to study dust properties of massive star forming regions in the outer Galaxy, in a direction opposite to the Galactic center.

Methods. We present observations of six outer Galaxy point sources IRAS 01045+6505, 01420+6401, 05271+3059, 05345+3556, 20222+3541 and 20406+4555, taken with the Submillimeter Common-User Bolometer Array (SCUBA) on the James Clerk Maxwell Telescope (JCMT) at 450 and $850 \mu \mathrm{m}$. Single temperature greybody models are fitted to the Spectral Energy Distribution of the detected sub-mm cores to derive dust temperature, dust emissivity index and optical depth at $250 \mu \mathrm{m}$. The observed radial intensity profiles of the sub-mm cores were fitted with power laws to derive the indices describing the density distribution.

Results. At a resolution of $15^{\prime \prime}$ all six IRAS point sources show multiple emission peaks. Only four out of fourteen detected sub-mm cores show associated mid-infrared emission. For the sub-mm cores we derive dust temperatures of $32 \pm 5 \mathrm{~K}$ and dust emissivity indices between 0.9 and 2.5 . The density profiles of the sub-mm cores can be fitted by a single power law distribution with indices $-1.5 \pm 0.3$, with most cores showing an index of -1.5 . This is consistent with most observations of massive star forming regions and supports predictions of models of star formation which consider non-thermal support against gravitational collapse.
\end{abstract}

Key words. stars: formation - ISM: general - ISM: H II regions - ISM: dust, extinction - submillimeter

\section{Introduction}

With the development of high angular resolution millimeter $(\mathrm{mm})$ and sub-millimeter (sub-mm) instrumentation, observations studying the influence of different physical environment on the formation of molecular clouds subsequently developing into protostellar cores has been receiving increased attention. While the inner Galaxy has been extensively studied, lack of instruments with high angular resolution has limited the study of sources in the outer Galactic disk which are in a potentially different physical and chemical environment. Existing observations suggest that in the outer Galactic disk the molecular clouds are more sparsely distributed (Wouterloot et al. 1990), the diffuse galactic interstellar radiation field is weaker (Cox \& Mezger 1989; Bloemen 1985), the metallicity is lower (Shaver et al. 1983; Fich \& Silkey 1991; Rudolph et al. 2006) and the cosmicray flux density is smaller (Bloemen et al. 1984), compared to the solar neighbourhood. Although the efficiency at which molecular clouds form from atomic gas is much lower in the far outer Galaxy, the mass spectrum of molecular clumps is not significantly different from the inner Galaxy (Snell et al. 2002). The spectra however show a tendency towards steepening in the far outer Galaxy. The star formation activity in the outer Galaxy clouds is also similar to the inner Galaxy clouds. Thus the observed low global star formation rate in the outer Galaxy is a consequence of the inefficiency of the formation of molecular clouds in this region and not an inefficiency in the star formation process within these clouds (Snell et al. 2002). The outer Galaxy star forming regions could thus be better examples of stars and/or stellar clusters forming in isolation in contrast to the crowded fields within the inner Galaxy. It is thus important to study the point sources identified by IRAS in the outer Galactic disk at high angular resolutions to probe their multiplicity, dust properties and kinematics. As a first step towards these goals we present here sub-mm continuum mapping of selected outer Galaxy IRAS sources.

We have selected a sample of outer Galaxy (with Galactocentric distances greater than $10 \mathrm{kpc}$ ) sources from the IRAS point source catalog (PSC) based on the following criteria: $F_{100} \mu \mathrm{m} \geq 100 \mathrm{Jy}, F_{100} \mu \mathrm{m}>F_{60} \mu \mathrm{m}, 30^{\circ} \leq l \leq 330^{\circ}$, $-5^{\circ} \leq b \leq 5^{\circ}$. The Galactic longitude criterion ensures that source confusion along the line of sight faced towards the inner Galaxy, is avoided. The sample consists of 17 sources, all of which are bona fide point sources according to the IRAS PSC. For majority of these sources no continuum maps other than the IRAS images are available. Here we present sub-mm (450 and $850 \mu \mathrm{m}$ ) observations of six sources in our sample, viz., IRAS 01045+6505, IRAS 01420+6401, IRAS 05271+3059, $05345+3556,20222+3541$, and $20406+4555$. For the sources IRAS $01045+6505$ and IRAS $01420+6401$ we have only $850 \mu \mathrm{m}$ observations, hence in this paper, we present only the basic results for these two sources and discuss the other four sources in detail.

The purpose of these observations was primarily to obtain a higher angular resolution view in order to detect the substructures within these sources. Using these observations we also derive the dust properties of these regions in order to improve our understanding of the true nature of sources located in the outer Galaxy environments. 


\section{Observations and data reduction}

We present here results of observations made between September 2004 and January 2005, using the Submillimeter Common-User Bolometer Array (SCUBA) on the James Clerk Maxwell Telescope (Holland et al. 1999). The SCUBA array covers a hexagonal 2.5 field of view with 91 and 37 pixels at 450 and $850 \mu \mathrm{m}$ respectively. Maps are fully sampled using the "jiggle" mode, in which the telescope is moved around a 64-position pattern by the secondary mirror in order to fully sample the sky with the wider intrinsic beam spacing of the array. A chop throw of $120^{\prime \prime}$ in azimuth was used for all observations.

The data were reduced using the SCUBA User Reduction Facility (SURF; Jenness \& Economou 1999) and the STARLINK imaging software following the methods described in the SCUBA mapping cookbook (Sandell et al. 2001). The steps of reduction included flat-fielding, extinction correction, sky-subtraction and calibration of images in $\mathrm{Jy} \mathrm{beam}^{-1}$. The maps were calibrated using observations of primary calibrators like Uranus, CRL 618 and CRL 2688 observed close to the time of our observations.

All the maps were converted to FITS-files and exported to MIRIAD (Sault et al. 1995) for further analysis. In order to correct for the error lobe contribution, we deconvolved all the maps using CLEAN and a symmetry-sized model beam. The model beam, derived from the 450 and $850 \mu \mathrm{m}$ observations of Uranus obtained during the same night and under similar conditions, and consistent with repeated observations during the whole observing run, is composed of three symmetric Gaussians. At $850 \mu \mathrm{m}$ it has HPBWs of 15'.2, 58.' 6 and 140", with amplitudes 0.985 , 0.013 and 0.002 , respectively. At $450 \mu \mathrm{m}$ it has HPBWs of 8.'5, $33^{\prime \prime}$ and $140^{\prime \prime}$ with amplitudes $0.942,0.055$ and 0.003 respectively. In order to obtain images with good $\mathrm{S} / \mathrm{N}$ we have restored maps at both 450 and $850 \mu \mathrm{m}$ to a common resolution of $15^{\prime \prime}$.

\section{Results}

Figure 1 shows the 450 and $850 \mu \mathrm{m}$ dust continuum maps of the six outer Galaxy IRAS point sources. We find that all the six sources are resolved into at least two to three emission peaks in the sub-mm images. Comparison with the MSX point source catalog suggests that most of the sub-mm cores do not have any associated MSX source. Given the $4^{\prime \prime}$ positional accuracy of the SCUBA maps (see below), we consider a sub-mm core to be associated with an MSX source only if the MSX source is within 5 " of the position of the sub-mm core. Given the large distances to the sources, displacement of the young star from its core due to proper motion outside of this association range is rather unlikely. Our interpretation is that the lack of MSX counterparts for the sub-mm sources is due to differences in the evolutionary stages. Based on this criterion we find that only 05271-SMM1, 05271-SMM2 and 01045-SMM1 have associated MSX sources. Only IRAS 01045+6505 was observed using IRAC and MIPS instruments on Spitzer. Preliminary results from Spitzer show that both 01045-SMM1 and 01045-SMM2 have sources at $24 \mu \mathrm{m}$ associated with them, with the IR source at the position of $01045-S M M 1$ being partially saturated. This indicates that $01045-\mathrm{SMM} 1$ is a reasonably hot source with a dust temperature of $40 \mathrm{~K}$ or more. IRAS $20406+4555,01420+6401$ and $01045+6505$ have associated radio continuum sources. For IRAS $01420+6401$ and $01045+6505$ the radio continuum positions are based on high resolution VLA observations (Rudolph et al. 1996), whereas for IRAS $20406+4555$ the radio data is from the GB6 catalog (Gregory et al. 1996). The nominal
IRAS position for $05345+3556$ is offset with respect to the ridge detected in the sub-mm. We have checked the ISSA images for this source and find that at the position given by the IRAS PSC there is no prominent peak. In Sect. 4 we discuss the sources individually in the light of the available limited complementary observations.

Given the extended nature of these sources and the $2^{\prime}$ chop throw, the observed off positions will not have been completely free of emission, and this will have been subtracted from the fluxes and as a background from the images. However, as we are more interested in the warmer dust corresponding to the warm cores seen by IRAS, rather than the cold diffuse component, the background-subtracted flux measurements are appropriate. In order to derive the parameters of the sub-mm cores detected in the IRAS sources, we have fitted an elliptical Gaussian for the sub-mm source, and a background plane for the surrounding cloud using the task IMFIT in MIRIAD. The fit to the broader component is mainly to provide a good subtraction of the extended emission, and is not to estimate the flux density of the surrounding cloud. Table 1 presents results of the fits to the detected sub-mm sources. We estimate the uncertainty in determining the positions of the sub-mm sources based on the Gaussian fitting to be $1^{\prime \prime}$. Combining this with the pointing accuracy of the observations $\left(<3^{\prime \prime}\right)$ we estimate the final astrometric uncertainty of the positions of the sub-mm cores to be $\sim 4^{\prime \prime}$. The sizes are derived from the Gaussian fits to the $450 \mu \mathrm{m}$ data and the flux densities at $850 \mu \mathrm{m}$ are derived by constraining the sizes of the Gaussian fits to be the same as the sizes at $450 \mu \mathrm{m}$. For the sub-mm cores not clearly detected at $450 \mu \mathrm{m}$, we have derived the sizes from the $850 \mu \mathrm{m}$ map and integrated the $450 \mu \mathrm{m}$ fluxes over an aperture of same size.

\section{Notes on the sources}

Here we present details of existing observations of the four sources IRAS $05271+3059,05345+3556,20222+3541$ and $20406+4555$.

IRAS 05271+3059: This source, WB89 651, is part of the outer Galaxy catalog compiled by Wouterloot \& Brand (1989). Based on the velocity information from the molecular line observations the distance to the source is estimated to be $16.5 \mathrm{kpc}$. The luminosity of the source estimated from the 60 and $100 \mu \mathrm{m}$ IRAS fluxes is $4.2 \times 10^{4} L_{\odot}$. CS(2-1) (Bronfman et al. 1996) and $\mathrm{H}_{2} \mathrm{O}$ (Valdettaro et al. 2001) maser emission was found to be associated with this source, although no methanol maser was detected (Slysh et al. 1999). There is no radio continuum source known to be associated with IRAS $05271+3058$. We find that both sub-mm sources are associated with MSX point sources (Fig. 1).

IRAS 05345+3556: Also known as WB89 673, this source is at a distance of $14 \mathrm{kpc}$ as derived from molecular line observations by Wouterloot \& Brand (1989) and has a luminosity of $1.7 \times 10^{4} L_{\odot}$. The radio continuum source detected in NRAO VLA Sky Survey (NVSS) at $1.4 \mathrm{GHz}$ (Condon et al. 1998) is coincident with the nominal position of the infrared peak according to the IRAS Point Source Catalog. As noted in Sect. 3 the dust emission detected in the SCUBA maps is along a ridge which is offset from this nominal infrared peak position. Similar to IRAS 05271+3059, CS(2-1) (Bronfman et al. 1996), SiO (Harju et al. 1998) and $\mathrm{H}_{2} \mathrm{O}$ (Valdettaro et al. 2001) maser emissions are found to be associated with this source and no methanol was detected (Slysh et al. 1999). No MSX point sources were found within a search radius of $20^{\prime \prime}$ around the sources SMM-1 and SMM-2, only SMM-3 has a nearby MSX source. 

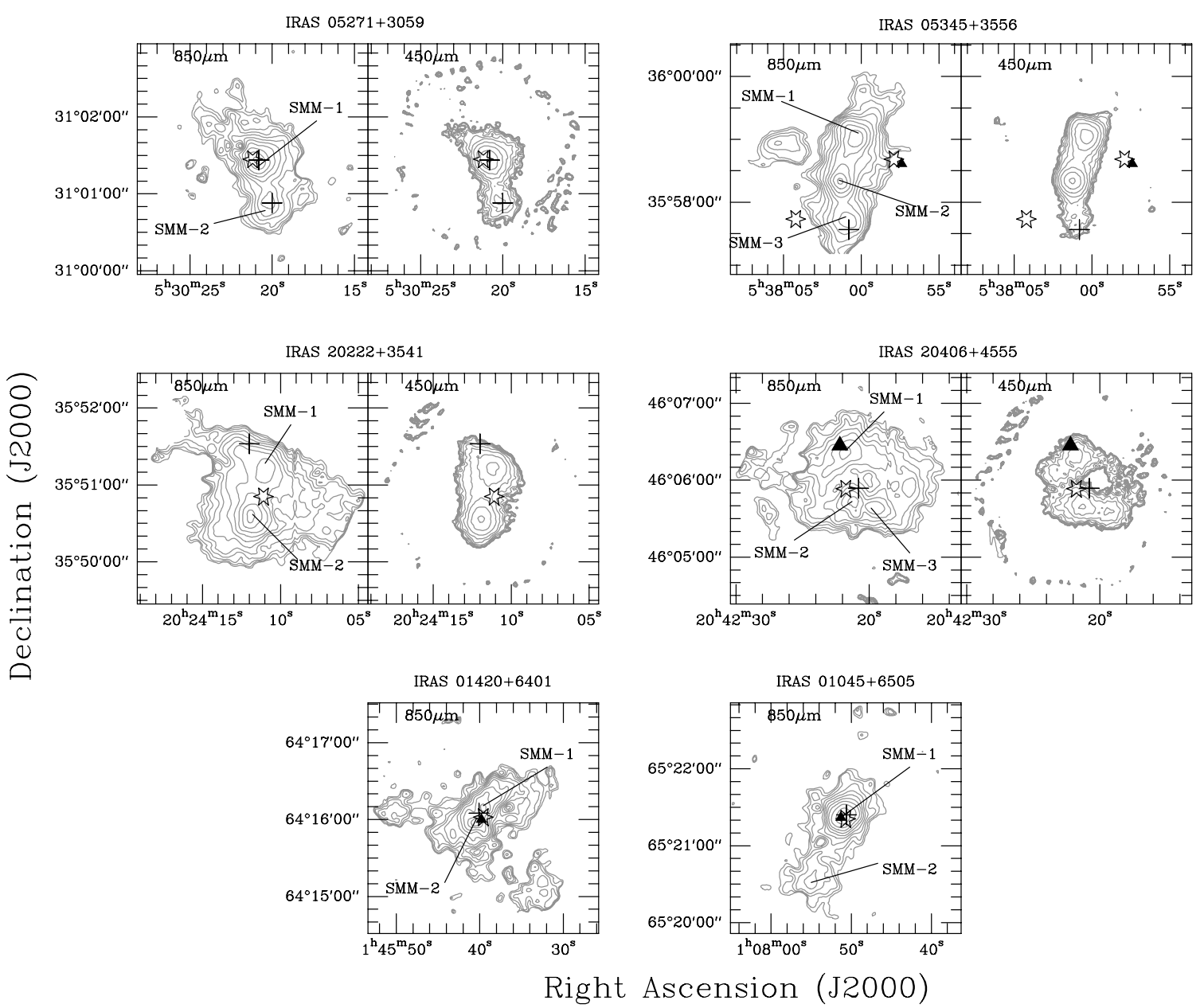

Fig. 1. Contours of 450 and $850 \mu \mathrm{m}$ emission for the outer Galaxy sources. "+" and $*$ indicate positions of MSX and IRAS point sources respectively in the mapped regions. Filled triangles show locations of radio continuum sources. The peak flux densities (rms) are as follows: (1) $05271+3059: F_{850}=0.6(0.02) \mathrm{Jy} / \mathrm{beam}, F_{450}=4.7(0.03) \mathrm{Jy} / \mathrm{beam} ;(2) 05345+3556: F_{850}=1.0(0.03) \mathrm{Jy} / \mathrm{beam}, F_{450}=8.0(0.04) \mathrm{Jy} / \mathrm{beam}$; (3) 20222+3541: $F_{850}=0.5(0.02) \mathrm{Jy} / \mathrm{beam}, F_{450}=3.2(0.02) \mathrm{Jy} / \mathrm{beam}$; (4) 20406+4555: $F_{850}=0.5(0.02) \mathrm{Jy} / \mathrm{beam}, F_{450}=1.8(0.01) \mathrm{Jy} / \mathrm{beam}$; (5) $01420+6401: F_{850}=0.4(0.02)$; (6) $01045+6505: F_{850}=1.2(0.03) \mathrm{Jy} /$ beam. 11 equally spaced logarithmic contours are drawn, with the lowest level at $2 \sigma$ and the highest level at $95 \%$ of the peak value.

IRAS 20222+3541: Beck \& Brosch (1988) suggested that IRAS 20222+3541, also known as MCG 06-45-001, is a local group galaxy. In a subsequent paper, Shore \& Sage (1990) used $\mathrm{CO}$ observations to conclusively show that rather than being an external galaxy, this IRAS source is a Galactic star forming region with an associated H II region. Based on the CO observations of Shore \& Sage (1990) we estimate the kinematic distance to the source to be $2.2 \mathrm{kpc}$ for a $v_{\text {lsr }}$ of $\sim 7.4 \mathrm{~km} \mathrm{~s}^{-1}$. This is the nearest of the sources in our sample. The luminosity of the source is $1.5 \times 10^{3} L_{\odot}$. Only one point source from the MSX catalog is found in the mapped region although the images of almost all MSX bands show an extended emission coincident with the IRAS source. The only identified MSX point source in this region is offset from SMM-1 by $\sim 25^{\prime \prime}$. In contrast to Shore \& Sage (1990) we find that the only radio continuum source detected in the NRAO VLA Sky Survey (NVSS) at $1.4 \mathrm{GHz}$ (Condon et al. 1998 ) is offset by $\sim 2^{\prime}$ with respect to the dust continuum peaks seen in IRAS, MSX and SCUBA datasets. In

IRAS 20406+4555: This source, also known as WB89 4 from the catalog of outer Galaxy sources by Wouterloot \& Brand (1989), is located at a distance of $7.8 \mathrm{kpc}$. We have confirmed from our recently concluded and yet unpublished $\mathrm{CO}$ observations, as well as from the ${ }^{13} \mathrm{CO} J=1-0$ observations of
Wu et al. (2001) that only the velocity component at $-49 \mathrm{~km} \mathrm{~s}^{-1}$ is associated with this source and this corresponds to the aforementioned distance. The luminosity of the source based on the IRAS fluxes is $2.6 \times 10^{4} L_{\odot}$. There are no MSX point sources in the MSX6C associated with this region, although both in bands $\mathrm{A}$ and $\mathrm{E}$ the source is clearly detected. An H II region from the GB6 catalog of radio sources (Gregory et al. 1996) is centerd at the position of SMM-1, while the IRAS source is located closer to SMM-2. The comparatively compact appearance of SMM- 2 \& 3 together with the $\mathrm{H}$ II region might suggest that SMM-2/3 contain protostars, formation of which is triggered by the $\mathrm{H}$ II region. However so far no $\mathrm{NH}_{3}$ and $\mathrm{H}_{2} \mathrm{O}$ maser emission were detected from this source (Molinari et al. 1996).

\section{Spectral energy distributions of the sub-mm cores}

Several authors (Sridharan et al. 2002; Beuther et al. 2002) have shown that two temperature components are present in the SED of luminous YSOs: a compact hot component which dominates the IRAS $12 \mu \mathrm{m}$ and $25 \mu \mathrm{m}$ fluxes, and a more extended component arising from colder gas which dominates the IRAS $60 \mu \mathrm{m}$ and $100 \mu \mathrm{m}$ fluxes. Here we analyze the spectral energy 
Table 1. Observed properties of the sub-mm sources identified in the SCUBA maps.

\begin{tabular}{ccccccccc}
\hline \hline $\begin{array}{c}\text { Submillimeter } \\
\text { source }\end{array}$ & $\alpha(2000)$ & $\delta(2000)$ & $\begin{array}{c}D \\
\mathrm{kpc}\end{array}$ & $\begin{array}{c}\theta_{a} \times \theta_{b}{ }^{a} \\
\left({ }^{\prime \prime} \times{ }^{\prime \prime}\right)\end{array}$ & $\begin{array}{c}S_{450} \\
\text { Jy }\end{array}$ & $\begin{array}{c}S_{850} \\
\text { Jy }\end{array}$ & $\begin{array}{c}\text { IR } \\
\text { Assoc. }\end{array}$ & $\begin{array}{c}\text { Radio } \\
\text { Assoc. }\end{array}$ \\
\hline $05271+3059$ SMM-1 & $05: 30: 20.97$ & $31: 01: 25.8$ & 16.5 & $12.9 \times 12.8$ & 7.92 & 1.08 & MSX & $\ldots$ \\
$05271+3059$ SMM-2 & $05: 30: 20.28$ & $31: 00: 50.3$ & 16.5 & $15.1 \times 12.7$ & 2.26 & 0.24 & MSX & $\ldots$ \\
\hline $05345+3556$ SMM-1 & $05: 38: 00.41$ & $35: 59: 00.2$ & 14.0 & $19.2 \times 14.1$ & 14.18 & 1.65 & $\ldots$ & $\ldots$ \\
$05345+3556$ SMM-2 & $05: 38: 01.29$ & $35: 58: 21.2$ & 14.0 & $13.9 \times 9.2$ & 12.74 & 1.14 & $\ldots$ & $\ldots$ \\
$05345+3556$ SMM-3 & $05: 38: 00.99$ & $35: 57: 45.7$ & 14.0 & $17.7 \times 14.5$ & $b$ & 0.80 & $\ldots$ & $\ldots$ \\
\hline $20222+3541$ SMM-1 & $20: 24: 10.69$ & $35: 51: 10.2$ & 2.2 & $20.4 \times 20.0$ & 6.06 & 0.33 & $\ldots$ & $\ldots$ \\
$20222+3541$ SMM-2 & $20: 24: 11.85$ & $35: 50: 34.8$ & 2.2 & $15.0 \times 13.2$ & 5.95 & 0.51 & $\ldots$ & $\ldots$ \\
\hline $20406+4555$ SMM-1 & $20: 42: 21.75$ & $46: 06: 21.1$ & 7.8 & $15.4 \times 11.0$ & 1.79 & 0.17 & $\ldots$ & GB6 \\
$20406+4555$ SMM-2 & $20: 42: 21.30$ & $46: 05: 42.8$ & 7.8 & $12.7 \times 12.6$ & 3.02 & 0.30 & $\ldots$ & $\ldots$ \\
$20406+4555$ SMM-3 & $20: 42: 19.33$ & $46: 05: 38.3$ & 7.8 & $15.7 \times 11.3$ & 2.17 & 0.24 & $\ldots$ & $\ldots$ \\
\hline $01420+6401-S M M 1$ & $01: 45: 39.09$ & $64: 16: 15.9$ & 10.1 & $14.8 \times 10.8$ & $\ldots$ & 0.44 & $\ldots$ & GB6,VLA \\
$01420+6401-S M M 2$ & $01: 45: 40.76$ & $64: 15: 57.3$ & 10.1 & $16.5 \times 16.3$ & $\ldots$ & 0.71 & $\ldots$ & $\ldots$ \\
\hline $01045+6505-S M M 1$ & $01: 07: 50.99$ & $65: 21: 23.2$ & 11.3 & $12.7 \times 11.6$ & $\ldots$ & 1.81 & MSX,Spitzer & GB6,VLA \\
$01045+6505-S M M 2$ & $01: 07: 54.99$ & $65: 20: 31.7$ & 11.3 & $16.7 \times 16.5$ & $\ldots$ & 0.46 & Spitzer & $\ldots$ \\
\hline
\end{tabular}

${ }^{a}$ Deconvolved Major $\left(\theta_{a}\right)$ and Minor $\left(\theta_{b}\right)$ axes derived from elliptical Gaussian fits. ${ }^{b}$ Not detected at $450 \mu \mathrm{m} . S_{450}$ obtained by integrating at the position of the $850 \mu \mathrm{m}$ peak is $5.29 \mathrm{Jy}$.

distributions (SED) of the sub-mm cores detected at both SCUBA wavelengths, between 60 and $850 \mu \mathrm{m}$ in order to derive order of magnitude estimates of the dust temperature and mass of the cold component. Since the sub-mm cores are not resolved in the mid and far-infrared, we apportion the 60 and $100 \mu \mathrm{m}$ flux densities for the IRAS sources to the sub-mm cores in the same ratio as the observed $450 \mu \mathrm{m}$ fluxes. 1998),

The greybody functions have the form (e.g., Dent et al.

$F_{v}=\Omega B_{v}\left(T_{\text {dust }}\right)\left(1-\mathrm{e}^{-\tau_{v}}\right)$,

where $F_{v}$ is the flux measured at a frequency $v, \Omega$ is the solid angle subtended by the cloud, $B_{v}\left(T_{\text {dust }}\right)$ is the Planck function evaluated at the dust temperature $T_{\mathrm{d}}$ and $v$, and $\tau_{v}$ is the optical depth at $\nu$. The optical depth $\tau_{v}$ scales as $\tau_{0} \nu^{\beta}$, where $\beta$ is the grain emissivity index and $\tau_{0}$ is the optical depth at a reference frequency $\left(v_{0}\right)$.

Fitting was performed such that $\chi^{2}$ was minimized, with $\chi^{2}$ defined as,

$\chi^{2}=\sum\left[1-\left(\frac{F_{v, \text { model }}}{F_{v, \text { obs }}}\right)\right]^{2}$.

This definition of $\chi^{2}$ gives equal weighting to the different wavelength regimes (Hunter et al. 2000). We have constrained the angular sizes of the sub-mm cores to those measured in the SCUBA observations (Table 2). Three free parameters were used: the temperature, the optical depth at $250 \mu \mathrm{m}$ and the grain emissivity index $(\beta)$.

Figure 2 shows the greybody models fitted to the SEDs and Table 2 also presents the parameters like mass, luminosity etc. calculated for the best fit model. The mass has been estimated from the fitted values of $\tau_{250}, T_{\mathrm{d}}$ and $\beta$, adopting the "Hildebrand" mass opacity, $\kappa_{\mathrm{o}}$, defined at $250 \mu \mathrm{m}(1200 \mathrm{GHz})$, i.e. $\kappa_{1200 \mathrm{GHz}}=0.1 \mathrm{~cm}^{2} \mathrm{~g}^{-1}$ (Hildebrand 1983) and a gas-to-dust ratio of 100 . The main uncertainty in calculating the dust masses here lie in the use of inner Galaxy dust properties as well as gas-to-dust ratio for these outer Galaxy sources. Observationally there is evidence of reduced metallicity towards the outer Galaxy (Rudolph et al. 2006) and the dust-to-gas ratio depends almost linearly on metallicity (Boselli et al. 2002). The assumed gasto-dust ratio of 100 does not take the metallicity gradient into account. In addition, the calculated dust mass opacities depend
Table 2. Parameters of the SED models for the sub-mm cores.

\begin{tabular}{|c|c|c|c|c|c|c|}
\hline Source & $\begin{array}{l}\text { Dia } \\
\text { pc }\end{array}$ & $\begin{array}{l}T \\
\mathrm{~K} \\
\end{array}$ & $\tau_{250}$ & $\beta$ & $\begin{array}{c}L \\
L_{\odot}\end{array}$ & $\begin{array}{c}M \\
M_{\odot}\end{array}$ \\
\hline$\overline{05271-S M M 1}$ & 1.03 & 36.9 & 0.01 & 1.2 & 42272 & 711 \\
\hline 05271-SMM2 & 1.10 & 28.6 & 0.01 & 1.9 & 15184 & 814 \\
\hline 05345-SMM1 & 1.12 & 30.7 & 0.01 & 0.9 & 13192 & 595 \\
\hline 05345-SMM2 & 0.77 & 28.6 & 0.03 & 1.2 & 12270 & 778 \\
\hline 05345-SMM3 & 1.09 & 28.6 & 0.01 & 1.0 & 6477 & 398 \\
\hline 20222-SMM1 & 0.22 & 26.6 & 0.02 & 2.5 & 1091 & 86 \\
\hline 20222-SMM2 & 0.15 & 28.6 & 0.03 & 2.2 & 1083 & 61 \\
\hline 20406-SMM1 & 0.49 & 28.6 & 0.01 & 2.4 & 6448 & 320 \\
\hline 20406-SMM2 & 0.48 & 30.7 & 0.02 & 2.4 & 12463 & 422 \\
\hline 20406-SMM3 & 0.50 & 32.8 & 0.01 & 2.4 & 15387 & 334 \\
\hline 01420-SMM1 & . & $32^{a}$ & $\ldots$ & $\ldots$ & $\ldots$ & $1041^{b}$ \\
\hline 01420-SMM2 & - & $32^{a}$ & . & & & $350^{b}$ \\
\hline 01045-SMM1 & . & $40^{a}$ & . & & & $267^{b}$ \\
\hline 01045-SMM2 & .. & $32^{a}$ & & & & $432^{b}$ \\
\hline
\end{tabular}

${ }^{a}$ Assumed $T_{\mathrm{d}} \cdot{ }^{b}$ Mass calculated from $S_{850}$ assuming the $T_{\mathrm{d}}$ given in the table and dust emissivity index $\beta=2$.

on the grain composition and even for inner Galaxy sources the commonly used values differ by as much as factors of 3 . Overall, we estimate that the calculated mass can deviate from the real value by up to a factor of 10 . For the sub-mm sources in IRAS $01045+6505$ and $01420+6401$ in the absence of the $450 \mu \mathrm{m}$ fluxes greybody SED fitting was not possible. We have estimated the mass of these sub-mm cores assuming $T_{\mathrm{d}}$ to be $32 \mathrm{~K}$, average value of $T_{\mathrm{d}}$ found from the SED fits and $\beta=2$. As discussed in Sect. 6 a choice of $\beta=2$ can be reasonably justified based on both theoretical and observational results.

Except for the sub-mm cores in IRAS 05345+3556, the greybody models produce reasonable fits to the SEDs of the sub-mm cores in IRAS $05271+3059,20222+3541$ and $20406+4555$. The SED models for the cores in IRAS $05345+3556$ do not reproduce the $450 \mu \mathrm{m}$ fluxes. A somewhat less constrained two dust temperature greybody model fit to the SED of the sub-mm cores in IRAS $05345+3556$ yields better fits. This indicates that additional observations between 100 and $450 \mu \mathrm{m}$ are essential to fully constrain the fits to the SEDs. 

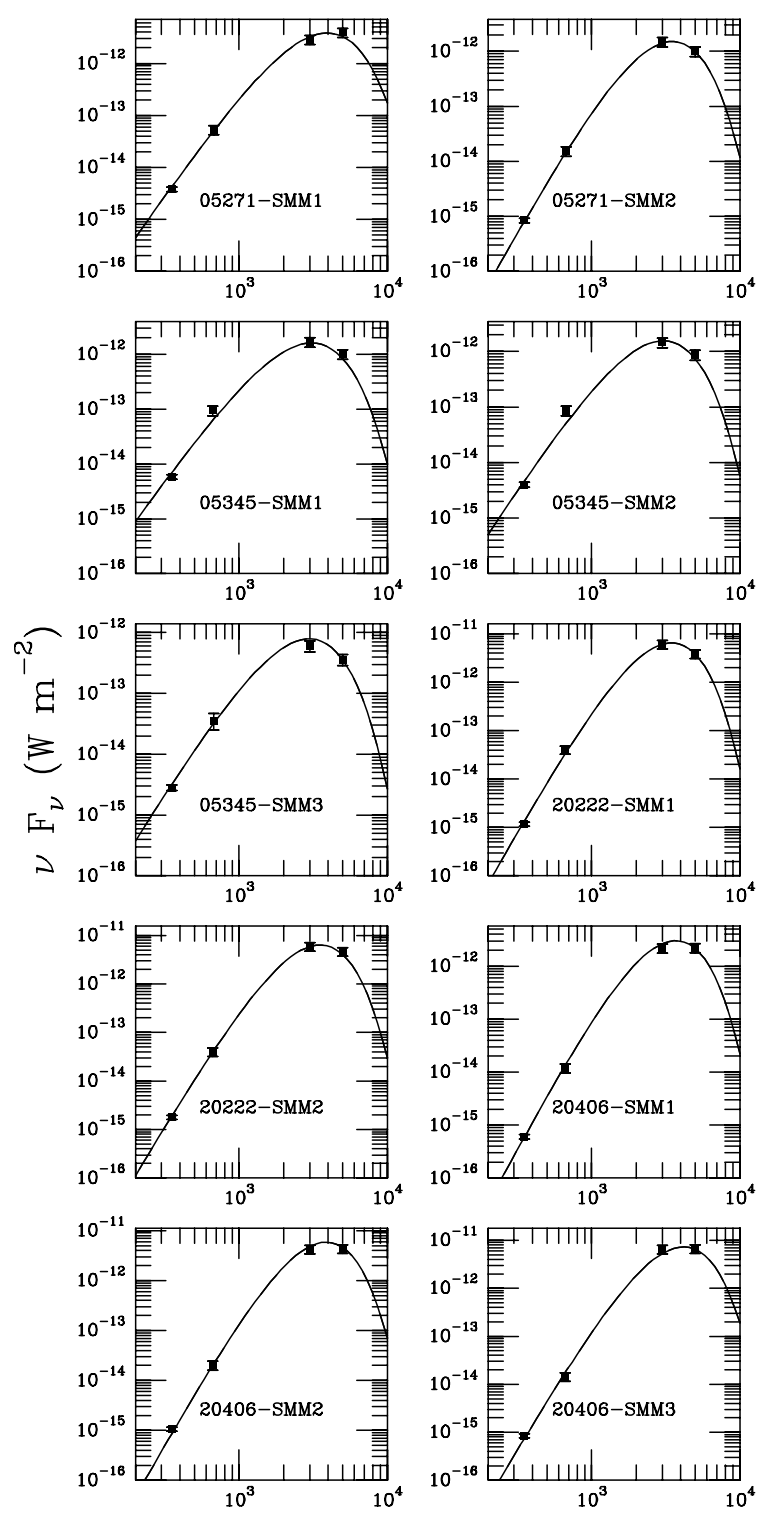

Frequency (GHz)

Fig. 2. Single temperature greybody fits to the spectral energy distribution (SED) of sub-mm cores. Fitted parameters are presented in Table 2.

\section{Dust properties from the SED fits}

The temperatures of the cold components for all the sub-mm cores are $\sim 32 \pm 5 \mathrm{~K}$ and the dust emissivity indices vary between 0.9 and 2.5. While the sub-mm cores within IRAS $05271+3059$ show very different dust emissivity indices ( $\beta$; Table 2$)$, the three sub-mm cores in IRAS $20406+4555$ show almost identical values of $\beta$.

The dust grain emissivity index is crucial in determining many source parameters, including the dust temperature, as well as providing information about the grain structure and the dielectric characteristics of the grains (Schwartz 1982; Hildebrand 1983). Knowledge of $\beta$ improves estimates of the dust opacity and subsequently of the dust mass of the star forming clouds. Various estimates of $\beta$ from both laboratory and theoretical models have been proposed and so far there is little consensus between these studies. Draine \& Lee (1984) derived $\beta \sim 2$ for $40 \leq \lambda \leq 1000 \mu \mathrm{m}$, using the available laboratory data to measure optical constants of a mixture of naked graphite and silicate grains. A dust grain emissivity index of $\sim 2$ has also been supported by the calculations of Knacke \& Thomson (1973), Mathis \& Whiffen (1989), Krügel \& Siebenmorgen (1994). Miyake \& Nakagawa (1993) however derived values of $\beta$ in excess of 2 for large grain sizes, while Wright (1987) calculated $0.6 \leq \beta \leq 1.4$ for fractal grains. Aannestad (1975) predicted $\beta$ up to 3.5 for olivine, fused quartz, and lunar rock grains covered with ice mantles. Mennella et al. (1995) concluded that more evolved cores have a higher emissivity index $(\beta)$.

Observationally there is an equally large scatter in the derived values of dust emissivity index, $\beta$, as in theoretical studies. There are several instances of observations of circumstellar disks around young PMS stars (Beckwith \& Sargent 1991; Sandell \& Weintraub 2001; Natta et al. 2004), low mass protostars (Hogerheijde \& Sandell 2000; Stark et al. 2004) as well as high mass protostars (Sandell 2000; Sandell \& Sievers 2004) for which $\beta<2$ were derived. Larger values of $\beta$, i.e., in excess of 2 have also been reported (Kuan et al. 1996; Goldsmith et al. 1997; Lis \& Menten 1998). We note here that the derived values of $\beta$ is always to some extent affected by the temperature and density variations as long as there is some optically thick portion of dust and detailed radiation transfer models can achieve a good fit to observations using $\beta=2$ (Mueller et al. 2002; Shirley et al. 2002). In addition, uncertainties and artifacts due to ignored error-lobe pickup, calibration uncertainties, optical thickness of H II regions, unequal beamsizes at the two wavelengths under consideration etc. have often contributed to observationally determined higher values of $\beta$.

The values of $\beta$ derived from the SCUBA observations of the outer Galaxy sources lie within the limits of the different values seen in the inner Galaxy sources. Based on the present estimates we conclude that the overall dust properties shown by the outer Galaxy sources, do not differ significantly from the properties of the inner Galaxy star forming regions, and show no indication of the substantially higher values of $\beta$ as found in a few extreme regions in the Galactic Center environment. The protostellar sources observed here are rather distant, hence the dust emission is dominated by the extended dense cloud envelopes surrounding the young high mass protostellar objects, rather than being dominated by the emission from the accretion disk, which are always found to show a lower apparent $\beta$.

\section{Radial profiles}

\subsection{Fits to the radial intensity profiles}

Figure 3 shows the radial intensity profiles at $850 \mu \mathrm{m}$ of all the sub-mm cores with the exception of 05345-SMM3 and 20406-SMM3. These radial profiles have been derived by averaging all datapoints within a $2^{\prime \prime}$ wide circular annulus at a radius $r$ from the center of the core, with $r$ ranging from 0 to $30^{\prime \prime}$. The observations were done with a chop throw of $2^{\prime}$ in the azimuth. Based on the derived angular sizes of the sub-mm cores we conclude that the effect of chopping on the derived radial intensity profiles is negligible.

We have created model intensity distribution by assuming an analytical two-dimensional power-law intensity distribution of the form $I(r) \propto \theta^{\gamma}$ and convolving it with the synthetic beam profile at $850 \mu \mathrm{m}$ which we used to deconvolve the intensity maps. We derived the model radial intensity profile by azimuthally averaging exactly in the same manner in which we have created the observed intensity profile. We have performed a $\chi^{2}$ fitting of a family of such model profiles corresponding to different power 


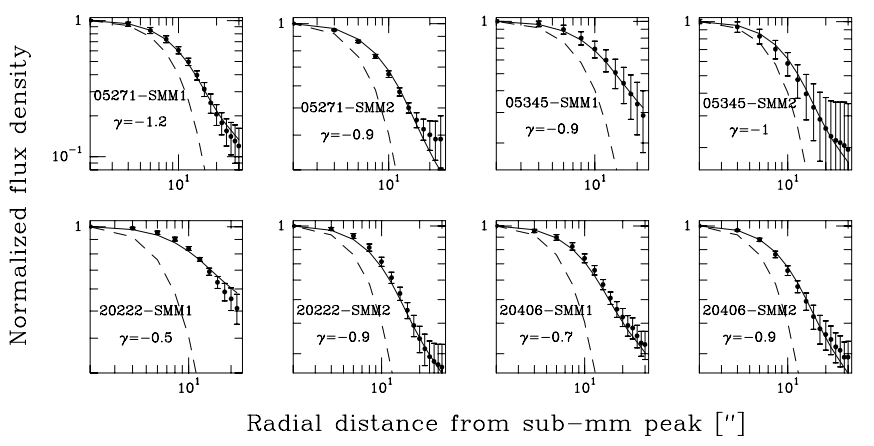

Fig. 3. Normalized, azimuthally averaged radial profiles for the intensities of the sub-mm cores at $850 \mu \mathrm{m}$ (points). Solid line shows the best fit power-law model corresponding to the index $\gamma$. The dashed line shows the model beam profile. The errorbars indicate the rms values within the annuli.

Table 3. Power law indices for radial intensity and density profile.

\begin{tabular}{ccc}
\hline \hline Source & $\gamma$ & $p$ \\
\hline 05271-SMM1 & -1.2 & -1.8 \\
05271-SMM2 & -0.9 & -1.5 \\
05345-SMM1 & -0.9 & -1.5 \\
05345-SMM2 & -1.0 & -1.6 \\
20222-SMM1 & -0.5 & -1.1 \\
20222-SMM2 & -0.9 & -1.5 \\
20406-SMM1 & -0.7 & -1.3 \\
20406-SMM2 & -0.9 & -1.5 \\
\hline
\end{tabular}

law indices to the observed radial profile. Keeping in mind the angular resolution of the $850 \mu \mathrm{m}$ images we have restricted the fitting procedure to radii $8^{\prime \prime}<r<30^{\prime \prime}$, so as to have a reliable estimate of the nature of the intensity distribution at spatial scales unaffected by the resolution of the observations. Figure 3 shows the resultant fits and Table 3 presents the fitted values of $\gamma$.

For most of the cores it is possible to derive acceptable fits to the radial intensity profiles of the outer envelopes $\left(r>8^{\prime \prime}\right)$ using a single power law. The fits, though not constrained by the inner regions, are reasonably good at radii $<8^{\prime \prime}$ as well and we derive $\gamma=-0.9 \pm 0.3$ for the 8 sub-mm cores.

\subsection{Density profiles of the sub- $\mathrm{mm}$ cores}

Here we consider a model with a spherically symmetric envelope and use the intensity distribution of the dust emission in the SCUBA images at $850 \mu \mathrm{m}$ to derive a first estimate of the radial density profile in the envelope. The density profile is a power law, $n \propto r^{p}$, the value of $p$ derived using this method depending upon the assumed temperature distribution.

For optically thin dust emission, assuming the Rayleigh-Jeans approximation to be valid, spherical cores and temperature and density distribution following a power law, the intensity index $\gamma$ depends to first order on the density index $p$ and the temperature index $q\left(T \propto r^{q}\right)$ through (Motte \& André 2001; Adams 1991):

$\gamma=1-p-q$.

Thus to derive the density index $p$ it is necessary to have some knowledge about the temperature distribution. Based on the grey-body fitting of the SEDs it is apparent that the sub-mm cores are centrally heated (accretion and/or stellar burning) so that the temperature decreases with increasing distance from the center. Theoretical calculations of massive star forming regions suggest that steeper indices for the temperature distribution are expected in the inner regions ( $<2000 \mathrm{AU})$. However beyond, the inner $2000 \mathrm{AU}$, the temperature distribution flattens again and runs asymptotically into a $r^{-0.4}$ distribution (van der Tak et al. 2000). For the large distances to our sources, the typical resolution of the $850 \mu \mathrm{m}$ maps are well beyond $20000 \mathrm{AU}$. This implies that we can safely assume the asymptotic solution of $q=0.4$ for our sources. Thus, for parts of the sub-mm cores at radii larger than $20000 \mathrm{AU}$ we derive density indices varying between -1.1 and -1.8 , with most cores showing indices of -1.5 (Table 3).

Analytic and numerical studies show that the mass infall rate during the protostellar phase is dependent on the radial density profile at the onset of collapse, and on the equation of state of the material. Motte \& André (2001) studied isolated and clustered protostellar envelopes, and found that isolated protostellar envelopes exhibit density profiles like $n \propto r^{-2}$, as predicted by the standard model of star formation by Shu et al. (1987). In contrast, protostellar envelopes in clusters are found to be induced in compact condensations resembling more finite-sized Bonnor-Ebert spheres than singular isothermal spheres, which suggests that dynamical protostellar models are more appropriate (Motte \& André 2001; Whitworth \& Summers 1985). ISO based mid-infrared absorption studies of low-mass prestellar cores revealed a flattening of the inner parts of the radial profiles as well as a steepening of the profiles farther outside (Bacmann et al. 2000). In the high mass regime studies of ultracompact $\mathrm{H}$ II regions and/or hot cores indicate that the overall density profiles are more like $n \propto r^{p}$ with $-1.0<p<-1.5$ as predicted by logatropic equations of state (Hatchell et al. 2000; van der Tak et al. 2000). Based on the study of a large sample of high mass protostellar objects (HMPOs) Beuther et al. (2002) derived a much wider range of density indices $n \sim r^{-1.6 \pm 0.5}$. A possible physical interpretation of the flattened density profile for massive objects could be that in low-mass objects the support against gravitational collapse is thermal, while for massive objects nonthermal support plays a major role (Myers \& Fuller 1992; McLaughlin \& Pudritz 1996).

Based on the luminosity estimates (Table 2) the sources we consider here are all intermediate to massive star forming regions, some of them having $\mathrm{H}$ II regions associated with them (Fig. 1). The derived value of density indices for the 8 sub$\mathrm{mm}$ cores is $-1.5 \pm 0.3$ and is thus consistent with observations of massive star forming regions and agree with theoretical models with significant non-thermal support against gravitational collapse.

\section{Summary}

The high angular resolution $\left(15^{\prime \prime}\right)$ sub-mm dust continuum maps of the outer Galaxy IRAS point sources show that all of them contain multiple emission peaks. Only four of the fourteen detected sub-mm sources have associated mid-infrared emission as seen from the MSX point source catalog and/or Spitzer IRAC and MIPS observations. SEDs for individual sub-mm cores between 60 and $850 \mu \mathrm{m}$ have been generated by apportioning the unresolved IRAS fluxes at 60 and $100 \mu \mathrm{m}$ in the same ratio as the observed $450 \mu \mathrm{m}$ fluxes. Single temperature greybody models were fitted to the observed SEDs of the sub-mm cores to derive the dust temperature, emissivity index $(\beta)$ and optical depth at $250 \mu$. The derived dust temperature of the sub$\mathrm{mm}$ cores is $32 \pm 5 \mathrm{~K}$ and the emissivity index ranges between 0.9 and 2.5. The two sub-mm cores in IRAS 05271+3059 show largely different emissivity indices. In spite of the known radial 
gradients in metallicity within the Milky Way, the dust properties derived here do not show any significant difference from the inner Galaxy star forming regions.

An analysis of the radial intensity profiles (for $r>8^{\prime \prime}$ ) at $850 \mu \mathrm{m}$ for the sub-mm cores shows that it is possible to fit the profiles reasonably well using a single power law with indices $-0.9 \pm 0.3$. Deriving density distributions from the radial indices we get density power laws $-1.5 \pm 0.3$, with most cores showing an index of -1.5 . This density profile is similar to the profile observed in many massive star forming regions in the inner Galaxy and generally agree with models of star formation which invoke non-thermal support against gravitational collapse (Myers \& Fuller 1992; McLaughlin \& Pudritz 1996).

Thus, except for their isolated occurence in the outer Galaxy, in most other respects the sources studied here look very simiilar to star formation cores in the inner parts of the Galaxy which have similar range of masses.

Acknowledgements. The James Clerk Maxwell Telescope is operated by The Joint Astronomy Centre on behalf of the Science and Technology Facilities Council of the United Kingdom, The Netherlands Organisation for Scientific Research, and the National Research Council of Canada. Data in this paper were obtained under the program ID M04BN04. This material is based upon work supported by the Deutsche Forschungs Gemeinschaft (DFG) via grant SFB494 and the National Science Foundation under Grant No. AST-0228974. This research has made use of NASA's Astrophysics Data System.

\section{References}

Aannestad, P. A. 1975, ApJ, 200, 30

Adams, F. C. 1991, ApJ, 382, 544

Bacmann, A., André, P., Puget, J.-L., et al. 2000, A\&A, 361, 555

Beck, S., \& Brosch, N. 1988, MNRAS, 232, 27

Beckwith, S. V. W., \& Sargent, A. I. 1991, ApJ, 381, 250

Bloemen, J. B. G. M. 1985, A\&A, 145, 391

Bloemen, J. B. G. M., Bennett, K., Bignami, G. F., et al. 1984, A\&A, 135, 12

Boselli, A., Lequeux, J., \& Gavazzi, G. 2002, A\&A, 384, 33

Bronfman, L., Nyman, L.-A., \& May, J. 1996, A\&AS, 115, 81

Beuther, H., Schilke, P., Menten, K. M., et al. 2002, ApJ, 566, 945

Condon, J. J., Cotton, W. D., Greisen, E. W., et al. 1998, AJ, 115, 1693

Cox, P., \& Mezger, P. G. 1989, A\&ARv., 1, 49

Dent, W. R. F., Matthews, H. E., \& Ward-Thompson, D. 1998, MNRAS, 301, 1049

Draine, B. T., \& Lee, H. M. 1984, ApJ, 285, 89

Fich, M., \& Silkey, M. 1991, ApJ, 366, 107

Goldsmith, P. F., Bergin, E. A., \& Lis, D. C. 1997, ApJ, 491, 615

Gregory, P. C., Scott, W. K., Douglas, K., \& Condon, J. J. 1996, ApJS, 103, 427

Harju, J., Lehtinen, K., Booth, R. S., \& Zinchenko, I. 1998, A\&AS, 132, 211

Hatchell, J., Fuller, G. A., Millar, T. J., Thompson, M. A., \& Macdonald, G. H. 2000, A\&A, 357, 637
Hildebrand, R. H. 1983, Royal Astron. Soc. Quart. J., 24, 267

Hogerheijde, M. R., \& Sandell, G. 2000, ApJ, 534, 880

Holland, W. S., Robson, E. I., Gear, W. K., et al. 1999, MNRAS, 303, 659

Hunter, T. R., Churchwell, E., Watson, C., et al. 2000, AJ, 119, 2711

Jenness, T., \& Economou, F. 1999, Astronomical Data Analysis Software and Systems VIII, ASP Conf. Ser., 172, 171

Knacke, R. F., \& Thomson, R. K. 1973, PASP, 85, 341

Krügel, E., \& Siebenmorgen, R. 1994, A\&A, 288, 929

Kuan, Y.-J., Mehringer, D. M., \& Snyder, L. E. 1996, ApJ, 459, 619

Lis, D. C., \& Menten, K. M. 1998, ApJ, 507, 794

Mathis, J. S., \& Whiffen, G. 1989, ApJ, 341, 808

McLaughlin, D. E., \& Pudritz, R. E. 1996, ApJ, 469, 194

Mennella, V., Colangeli, L., \& Bussoletti, E. 1995, A\&A, 295, 165

Miyake, K., \& Nakagawa, Y. 1993, Icarus, 106, 20

Molinari, S., Brand, J., Cesaroni, R., \& Palla, F. 1996, A\&A, 308, 573

Motte, F., \& André, P. 2001, A\&A, 365, 440

Mueller, K. E., Shirley, Y. L., Evans, N. J., II, \& Jacobson, H. R. 2002, ApJS, 143,469

Myers, P. C., \& Fuller, G. A. 1992, ApJ, 396, 631

Natta, A., Testi, L., Neri, R., Shepherd, D. S., \& Wilner, D. J. 2004, A\&A, 416, 179

Palla, F., Brand, J., Comoretto, G., Felli, M., \& Cesaroni, R. 1991, A\&A, 246, 24994

Rudolph, A. L., Brand, J., de Geus, E. J., \& Wouterloot, J. G. A. 1996, ApJ, 458, 653

Rudolph, A. L., Fich, M., Bell, G. R., et al. 2006, ApJS, 162, 346

Sandell, G. 2000, A\&A, 358, 242

Sandell, G., \& Weintraub, D. A. 2001, ApJS, 134, 115

Sandell, G., \& Sievers, A. 2004, ApJ, 600, 269

Sandell, G., Jessop, N., \& Jenness, T. 2001, The SCUBA map reduction Cookbook, Starlink Cookbook 11.2, Rutherford Appleton Laboratory, Particle Physics \& Astronomy Research Council

Sault, R. J., Teuben, P. J., \& Wright, M. C. H. 1995, Astronomical Data Analysis Software and Systems IV, ASP Conf. Ser., 77, 433

Schwartz, P. R. 1982, ApJ, 252, 589

Shaver, P. A., McGee, R. X., Newton, L. M., Danks, A. C., \& Pottasch, S. R. 1983, MNRAS, 204, 53

Shirley, Y. L., Evans, N. J., II, \& Rawlings, J. M. C. 2002, ApJ, 575, 337

Shore, S. N., \& Sage, L. J. 1990, ApJ, 362, L9

Shu, F. H., Adams, F. C., \& Lizano, S. 1987, ARA\&A, 25, 23

Slysh, V. I., Val'tts, I. E., Kalenskii, S. V., et al. 1999, A\&AS, 134, 115

Snell, R. L., Carpenter, J. M., \& Heyer, M. H. 2002, ApJ, 578, 229

Sridharan, T. K., Beuther, H., Schilke, P., Menten, K. M., \& Wyrowski, F. 2002, ApJ, 566, 931

Stark, R., Sandell, G., Beck, S. C., et al. 2004, ApJ, 608, 341

Valdettaro, R., et al. 2001, A\&A, 368, 845

van der Tak, F. F. S., van Dishoeck, E. F., Evans, N. J., II, \& Blake, G. A. 2000, ApJ, 537, 283

Whitworth, A., \& Summers, D. 1985, MNRAS, 214, 1

Wood, D. O. S., \& Churchwell, E. 1989, ApJ, 340, 265

Wouterloot, J. G. A., \& Brand, J. 1989, A\&AS, 80, 149

Wouterloot, J. G. A., Brand, J., Burton, W. B., \& Kwee, K. K. 1990, A\&A, 230, 21

Wright, E. L. 1987, ApJ, 320, 818

Wu, Y., Wu, J., \& Wang, J. 2001, A\&A, 380, 665 